\section{Revista de CIENCIAS AMBIENTALES Tropical Journal of Environmental Sciences}

Revista de Ciencias Ambientales (Trop J Environ Sci)

e-ISSN: 2215-3896

(Enero-Junio, 2022) . Vol 56(1): 268-283

DOI: https://doi.org/10.15359/rca.56-1.14

Open Access: www.revistas.una.ac.cr/ambientales e-mail: revista.ambientales@una.ac.cr

Jiménez Q.

\title{
EXPERIENCIAS \\ La gestación y culminación del Manual de Plantas de Costa Rica: una herramienta para el conocimiento y la conservación de la flora neotropical
}

\author{
The gestation and culmination of the Manual of Plants of Costa Rica: a tool for the \\ knowledge and conservation of the neotropical flora
}

\author{
Quírico Jiménez Madrigal ${ }^{1}$
}

\section{Resumen}

El Manual de Plantas de Costa Rica ha sido quizá la obra más importante en el campo de las ciencias naturales publicada en Costa Rica en las últimas décadas. Este proyecto iniciado a mediados de los años 80 del siglo anterior y concluido con su octavo volumen a finales del año 2020 tuvo como principal objetivo catalogar las plantas de Costa Rica, para lo cual se nutrió de una rica historia y de una intensa exploración botánica que nació hace varios siglos. Dos instituciones costarricenses, el Museo Nacional de Costa Rica y el Instituto Nacional de Biodiversidad, abrieron sus puertas al Jardín Botánico de Missouri, para que juntos pudieran consolidar y finalmente concluir con esta obra tan valiosa para el país, para la población costarricense y para toda la América tropical. En ella se describen las más de 9000 especies de plantas, ubicadas en 237 familias, con semilla que existen en el país, aunque hoy ya son más de 11000 especies si incluimos en ellas el grupo de los helechos. Esta alta diversidad de especies de plantas en un territorio tan pequeño convierte a Costa Rica en un país altamente biodiverso desde el punto de vista florístico.

Palabras clave: Biodiversidad; botánicos; Manual de Plantas; para-taxónomos

\begin{abstract}
The Manual of Plants of Costa Rica has been perhaps the most important work in the field of natural sciences published in Costa Rica in recent decades. This project, which started in the mid-80s of the previous century and concluded with its eighth volume at the end of 2020, had as its main objective to catalog the plants of Costa Rica, for which it was nourished by a rich history and an intense botanical exploration that was born several centuries ago. Two Costa Rican institutions, the National Museum of Costa Rica and the National Institute of Biodiversity, opened their doors to the Missouri Botanical Garden so that together they could consolidate and finally conclude this work so valuable for the country, for Costa Ricans and for all of Tropical America. It describes the more than 9 000 species of seed plants in 237 families that occur in the country, although today there are more than 11000 species if we include the group of ferns and their allies. This high diversity of plant species in such a small area makes Costa Rica a highly biodiverse country from the floristic point of view.
\end{abstract}

Keywords: biodiversity; botanists; Handbook of plants; para-taxonomists.

1 Funcionario de la Empresa de Servicios Públicos de Heredia (ESPH); profesor de la Universidad Técnica Nacional (UTN), Costa Rica; qjimenez@esph-sa.com

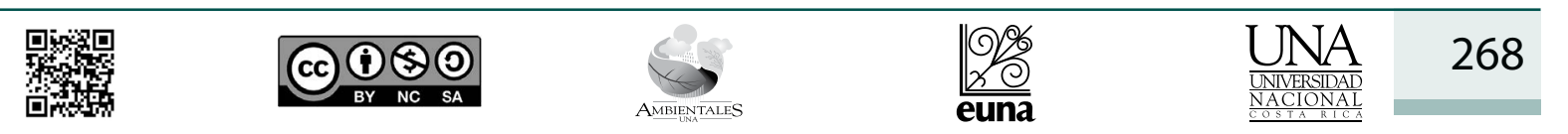




\section{Revista de CIENCIAS AMBIENTALES Tropical Journal of Environmental Sciences}

Revista de Ciencias Ambientales (Trop J Environ Sci)

e-ISSN: 2215-3896

(Enero-Junio, 2022) . Vol 56(1): 268-283

DOI: https://doi.org/10.15359/rca.56-1.14

Open Access: www.revistas.una.ac.cr/ambientales e-mail: revista.ambientales@una.ac.cr Jiménez Q.

\section{Introducción}

Difícilmente se puedan contar con lujo de detalles las peripecias y aventuras que ocurrieron en más de tres décadas, desde su inicio en 1987 hasta la culminación en el año 2020, de la extraordinaria obra Manual de Plantas de Costa Rica, compuesta por ocho volúmenes. Sin duda, un gran esfuerzo de los principales responsables y editores, los colegas Barry Hammel y Michael Grayum, funcionarios del Jardín Botánico de Missouri (Missouri Botanical Garden).

Hay tanto que contar, que se complica por dónde empezar. Quizá por esto creo que lo primero que debo hacer es dar un agradecimiento a nuestra Madre Natura, que con tantos siglos de evolución puso parte de su corazón en el pequeño terruño costarricense para convertirlo en un país biodiverso, rico en flora y en fauna, al cual a través del tiempo, según Hammel et al. (2004), en su estudio han participado - en el caso específico de las plantas - más de 175 profesionales en Botánica, entre nacionales y extranjeros, tomando como punto de partida la llegada al país del danés Anders S. Oersted en 1846.

Sin duda, la prodigiosa biodiversidad del país ha llamado la atención a propios y extraños, pero también no podemos dejar de lado una situación que es un sello costarricense y que de alguna manera es una gran bendición. Me refiero a que este país ha sido políticamente estable, a diferencia de los demás países centroamericanos, y aunque el estudio de las ciencias naturales al inicio estuvo más en manos de personas extranjeras, ya en el siglo anterior hubo muchos costarricenses que contribuyeron al estudio de nuestras plantas.

En este artículo no pretendo anotar todo lo que ya se conoce sobre el país, relacionado con la gran cantidad de profesionales en botánica que nos visitaron, sus estudios y publicaciones. Se anotarán algunos puntos en forma muy general, y ahondaré en aquellos que el lector desconoce, por tratarse de actividades y situaciones solo conocidas por las personas que participaron en las muchas actividades relacionadas con el proyecto del Manual, y quizá ignoradas.

Finalmente, es importante señalar que este proyecto del Manual será de gran ayuda no solo para la población costarricense, sino también para las personas extranjeras que nos visitan con fines turísticos o que vienen a estudiar nuestra biodiversidad, en especial nuestra flora. A través del tiempo, muchas personas en Costa Rica han dejado una huella indeleble, tratando de proteger esta diversidad biológica, de la cual hoy nos enorgullecemos. La lucha por la protección de esta biodiversidad ha consolidado el Sistema Nacional de Áreas de Conservación (SINAC), un trabajo que no ha sido fácil y hoy afortunadamente se ha ganado un gran reconocimiento a nivel mundial.

\section{La gran riqueza de Costa Rica: su biodiversidad}

Nuestro país se encuentra ubicado en un istmo relativamente joven en su formación, si lo comparamos con Norte y Suramérica. Su geología es demasiado compleja (Hammel et al., 2004). Varios procesos geológicos y volcánicos formaron este istmo, lo cual según Valerio (2006), ocurrió hace más de tres millones de años, en la época del Plioceno. Este istmo se formó a partir de sedimentos marinos y producto de una gran actividad tectónica y volcánica. Para Alvarado

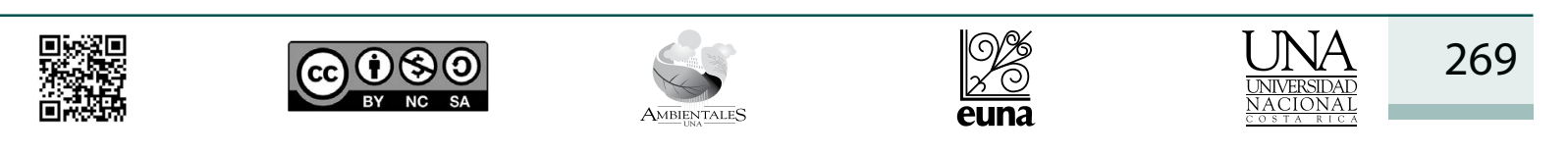




\section{Revista de CIENCIAS AMBIENTALES Tropical Journal of Environmental Sciences}

Revista de Ciencias Ambientales (Trop J Environ Sci)

e-ISSN: 2215-3896

(Enero-Junio, 2022) . Vol 56(1): 268-283

DOI: https://doi.org/10.15359/rca.56-1.14

Open Access: www.revistas.una.ac.cr/ambientales e-mail: revista.ambientales@una.ac.cr

Jiménez Q.

y Cárdenas (2016), Costa Rica es el resultado de un intrincado mosaico de paisajes dinámicos, formado por una amplia gama de procesos, como el vulcanismo, la tectónica, la erosión y deposición fluvial y marina, la meteorización y los procesos hidrotermales, kársticos, glaciales y periglaciales. El resultado es una provincia fisiográfica caracterizada como un conjunto distintivo de accidentes geográficos que contienen una historia única de evolución del paisaje.

Según Jiménez (1999), a pesar del pequeño tamaño de su territorio, Costa Rica posee una flora muy rica. Su diversidad climática, topográfica y edafológica, así como la influencia de dos océanos y una gran diversidad faunística, especialmente de insectos, y la cercanía con tres floras como lo son la de Norteamérica (especialmente México), la de Suramérica (región Amazónica y Cordillera de los Andes) y en menor grado la de las islas del Caribe, hacen de nuestro país un puente geográfico entre las dos Américas. Para Gámez et al. (2021) el fenómeno de su biodiversidad obedece sobre todo a su condición ístmica o de puente biológico o biogeográfico, entre dos inmensos subcontinentes que estuvieron separados desde tiempos inmemoriales.

Lo anterior ha dado como resultado que nuestro país, con apenas un $0,42 \%$ del territorio mundial, posea aproximadamente el $5 \%$ de la biodiversidad mundial, con un 3,5\% de todas las plantas conocidas en el planeta y estimadas en 300.000 especies, aunque un estudio más reciente (Ulloa et al., 2017) menciona un total de 383.671 especies de plantas vasculares conocidas en el mundo. No podemos ignorar, por su importancia, el endemismo florístico, que se estima en cerca de 1000 especies. Para Burger (1980) y Gómez (1986), esa compleja historia geológica, su variedad de climas y su heterogeneidad topográfica han convertido a Costa Rica en un caleidoscopio de ecosistemas terrestres y en el mejor representante de la eco-diversidad en América Central.

Adicionalmente, su notable variedad topográfica, con cordilleras que dividen el país en dos vertientes, algunas con volcanes activos, más su diversidad climática, han contribuido además al establecimiento de esa gran variedad de ecosistemas con una gran diversidad florística. Según Obando Acuña (2002) y Kappelle (2016), se estima que Costa Rica tiene el 5 \% de todas las especies oficialmente descritas en nuestro planeta. Esta información es conocida debido a la excelente colaboración al estilo del mejor mutualismo, que se ha tenido durante muchas décadas entre profesionales costarricenses y de otros países. Las plantas, sin duda, ocupan un espacio importante en esta cifra.

\section{Los primeros recolectores extranjeros}

Como se menciona en la introducción del primer volumen del Manual de Plantas (Hammel et al., 2004), este proyecto de catalogar las plantas de Costa Rica se ha nutrido de una rica historia y de una intensa exploración de la flora costarricense, como lo documentan en detalle León (2002) e Hilje (2013), de cuyos textos proviene la siguiente síntesis.

Los primeros registros de nuestra flora aparecen en los relatos del cronista español Gonzalo Fernández de Oviedo, en su obra Historia general y natural de las Indias, islas y tierra firme del mar océano; desde 1513, y durante 22 años, él recorrió Mesoamérica y el Caribe, incluyendo una parte de Costa Rica. Posteriormente, la Corona Española organizó dos grandes y prolongadas

\begin{tabular}{|c|c|c|c|c|c|}
\hline 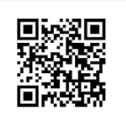 & (c) (i) (5) () & $\theta_{\text {AMBIENTALS }}^{6}$ & $\frac{10 \%}{\frac{9}{2}}$ & 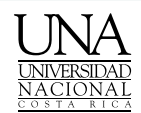 & 270 \\
\hline
\end{tabular}




\section{Revista de CIENCIAS AMBIENTALES Tropical Journal of Environmental Sciences}

Revista de Ciencias Ambientales (Trop J Environ Sci)

e-ISSN: 2215-3896

(Enero-Junio, 2022) . Vol 56(1): 268-283

DOI: https://doi.org/10.15359/rca.56-1.14

Open Access: www.revistas.una.ac.cr/ambientales e-mail: revista.ambientales@una.ac.cr

Jiménez Q.

expediciones a Nueva España (México). La primera (1571-1577) fue liderada por el médico, botánico y ornitólogo Francisco Hernández de Toledo, mientras que la segunda ocurrió dos siglos después (1787-1803), conducida por los médicos y botánicos Martín de Sessé Lacasta, Vicente Cervantes Mendo y José Mariano Mociño. Aunque se concentraron en México, hay evidencias indirectas de que Mociño hizo un breve recorrido entre Puntarenas a Cartago (León, 2002), por lo que a él se le deberían las primeras herborizaciones formales en Costa Rica.

Ya en la época independiente, entre 1836 y 1842, mientras realizaba mapeos de nuestras costas el barco inglés HMS Sulphur, al mando de Sir Edward Belcher, se aprovechó para recolectar plantas, lo cual estuvo a cargo del médico Andrew Sinclair y de George Barclay, quien era empleado del jardín botánico de Kew; los especímenes fueron remitidos al reputado taxónomo George Bentham. Sin embargo, estas recolecciones se efectuaron no muy lejos del litoral. El primero en realizar herborizaciones en el interior de Costa Rica fue el diplomático y naturalista austríaco Emanuel Ritter von Friedrichsthal, llegado por sus propios medios a mediados de 1839, pero partió pronto hacia Nicaragua y México.

Siete años después arribó por cuenta propia el zoólogo y botánico danés Anders S. Oersted, quien residió por casi año y medio en el país. A su regreso, basado en el material botánico recolectado en las islas Vírgenes y Jamaica - donde estuvo previamente-, así como en Costa Rica y Nicaragua, publicó 43 artículos científicos, al igual que el libro L’Amerique Centrale, con abundante información botánica sobre nuestro país, aunque a su regreso también recorrió Nicaragua; pensaba publicar unos 4 o 5 libros cortos, pero su muerte, a los 56 años, lo impidió. Cuando regresaba, en Nicaragua se topó con el naturalista polaco Josef von Warszewicz, quien había trabajado como botánico en una colonia belga instalada en Guatemala, pero después se independizó y se dedicó a recolectar orquídeas y otras plantas, así como anfibios, reptiles y colibríes, para venderlos a coleccionistas, museos y jardines botánicos en Europa.

Durante los siguientes seis años no hubo herborizaciones. No fue sino en 1854 que éstas se reanudaron, gracias a la presencia de los naturalistas alemanes Karl Hoffmann y Julián Carmiol, que se establecieron en el país, aunque el primero murió en 1859.

Asimismo, dicha actividad se intensificaría en 1874, con la llegada del también alemán Otto Kuntze a Puerto Limón, quien con fondos propios y durante tres semanas recorrió el país de costa a costa, para recolectar unos 600 especímenes. Sería sucedido por su compatriota Helmuth Polakowsky, quien estuvo apenas un año en el país, en 1875-1876, contratado como profesor de secundaria, en el Instituto Nacional, pero por sus propios medios recorrió gran parte del país. Prolífico como autor, a él se deben los siguientes artículos: Lista de briófitas y cormófitas de Costa Rica recogidas por H. Polakowsky en el año de 1875 (1877); Catálogo de las plantas costarricenses recogidas por $H$. Polakowsky en el año de 1875 (1877); Apuntes sobre la flora y la distribución de la vegetación de la República de Costa Rica (1877); Contribución para el conocimiento de la flora de Costa Rica en América Central (1877); Agricultura y horticultura de la República de Costa Rica, en América Central (1877); Contribución para el conocimiento de la distribución de las especies

\begin{tabular}{|c|c|c|c|c|c|}
\hline 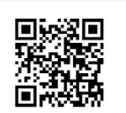 & (c) (1) (9) & $\underbrace{}_{\text {AMBENTIIES }}$ & $\frac{10 \%}{20}$ & $\frac{\text { UNA }}{\frac{\text { UNIVERSIDAD }}{\text { NACIONAL }}}$ & 271 \\
\hline
\end{tabular}




\section{Revista de CIENCIAS AMBIENTALES Tropical Journal of Environmental Sciences}

Revista de Ciencias Ambientales (Trop J Environ Sci)

e-ISSN: 2215-3896

(Enero-Junio, 2022) . Vol 56(1): 268-283

DOI: https://doi.org/10.15359/rca.56-1.14

Open Access: www.revistas.una.ac.cr/ambientales e-mail: revista.ambientales@una.ac.cr

Jiménez Q.

vegetales de Costa Rica, parte de América Central (1877); y La flora de Costa Rica. Contribución para el estudio de la fitogeografía centroamericana (1879).

No obstante, en realidad la época de mayor auge en herborizaciones ocurrió gracias a la creación, en 1889, del Instituto Físico-Geográfico Nacional, por iniciativa del geógrafo y botánico suizo Henri Pittier, quien en 1889 contrató a su compatriota Adolphe Tonduz como encargado del Hebario Nacional. Ambos recorrieron el país en largas y extenuantes jornadas, lo cual permitió que para 1904 hubiera 18.000 especímenes de plantas recolectadas. De esos esfuerzos surgió la obra cumbre Primitiae Florae Costaricensis, escrita junto con el taxónomo belga Théophile Durand, del Jardín Botánico de Bruselas, la cual constó de tres volúmenes y 12 fascículos, aparecidos entre 1891 y 1905. Cabe acotar que, después de residir 17 años en Costa Rica, en 1905 partió hacia Washington, para trabajar con el Departamento de Agricultura (USDA), mientras que Tonduz permaneció casi 31 años aquí. Durante esos años, llegó por cuenta propia el alsaciano Carlos Wercklé, cuyo mayor aporte botánico fue el extenso artículo La subregión fitogeográfica costarricense.

Después de estos grandes botánicos, las mayores contribuciones en el campo botánico provinieron del estadounidense Paul C. Standley, quien, especialmente con la ayuda del botánico Juvenal Valerio Rodríguez, funcionario del Museo Nacional, pudo recolectar unos 15.000 ejemplares de plantas, lo que entre 1937 y 1938 le permitiría publicar la extraordinaria obra Flora de Costa Rica, la cual consta de cuatro partes y abarcó 6085 especies.

Es pertinente indicar que, antes de Valerio habían realizado importantes aportes los jóvenes botánicos Otón Jiménez Luthmer, Alberto Manuel Brenes Mora y Juan José Cooper Sandoval, a quienes años después se les sumarían José María Orozco Casorla, Jorge León Arguedas y Rafael Lucas Rodríguez Caballero. Con ellos se había iniciado una tradición de colaboración internacional, que hizo posible que - como en el caso de Standley-, el Museo Nacional se convirtiera en el lugar natural para, con el paso de los años, atraer y acoger a tantos extranjeros interesados en explorar nuestra flora.

En tal sentido, sobresalió la obra Flora Costaricensis. Es oportuno destacar que este proyecto fue impulsado por el botánico Louis O. Williams, quien había trabajado con Standley en Honduras, cuando laboraba para el Field Museum of Natural History, en Chicago. Contó con el apoyo de varias becas de la Fundación Nacional de Ciencias de EE.UU., e inicialmente participaron los botánicos Antonio Molina y Alfonso Jiménez, en Honduras y Costa Rica, respectivamente. El primero de 13 volúmenes o fascículos, que abarcan 52 familias de plantas, data de 1971, y fue publicado por William C. Burger, el principal botánico del proyecto, mientras que el último apareció en 2010.

\section{El inicio del proyecto del Manual de Plantas}

Es importante destacar como uno de los principales inspiradores de este proyecto al Dr. Peter Raven, director en su momento del Jardín Botánico de Missouri, quien en 1986 solicitó la colaboración de los doctores Barry Hammel y Michael H. Grayum. Ellos, como botánicos de la

\begin{tabular}{|c|c|c|c|c|}
\hline 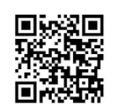 & (c) (1) () (2) & $\underset{\text { AMBENENALIES }}{8}$ & $\frac{10 \%}{\frac{\sigma}{2}}$ & 272 \\
\hline
\end{tabular}




\section{Revista de CIENCIAS AMBIENTALES Tropical Journal of Environmental Sciences}

Revista de Ciencias Ambientales (Trop J Environ Sci)

e-ISSN: 2215-3896

(Enero-Junio, 2022) . Vol 56(1): 268-283

DOI: https://doi.org/10.15359/rca.56-1.14

Open Access: www.revistas.una.ac.cr/ambientales e-mail: revista.ambientales@una.ac.cr

Jiménez Q.

misma institución, asumieron con responsabilidad esta importante iniciativa. En ese momento, Grayum había residido varios años en Costa Rica con su esposa Pamela Sleeper, al punto de que aquí nació su único hijo, Jeffrey. Luego, en 1989 llegó Hammel, quien aún vive en Costa Rica junto a su familia. Al año siguiente, Grayum volvió a Missouri a continuar con el proyecto desde allí, con visitas esporádicas al país. Por muchos años ambos realizaron expediciones a Costa Rica, a la vez que ayudaron en la identificación de especímenes recolectados por ellos y otros botánicos, tanto costarricenses como de otros países.

Desde que el proyecto del Manual afianzó sus raíces, en 1987, se pensó que su sede principal estuviera en Costa Rica y no en Missouri. Primero fue albergado por el Museo Nacional, y luego por el novel Instituto Nacional de Biodiversidad (INBio). Los objetivos para tal decisión fueron los siguientes (Hammel et al., 2004): estimular la creación aquí de bases de datos computarizadas, para tratar las colecciones de plantas realizadas; involucrar al máximo a los botánicos y para-taxónomos nacionales; y publicar la obra en español, como una guía ilustrada para la identificación de las plantas del país. Es de destacar el mutualismo que existió entre el Jardín Botánico de Missouri, el INBio y el Museo Nacional, para lograr la consolidación de este Manual.

El proyecto contó además con la ayuda económica de varias becas, aportadas por la National Science Foundation (NSF) de los Estados Unidos, así como por la National Geographic Society, con el objetivo de realizar expediciones a la vertiente caribe de la cordillera de Talamanca, la península de Santa Elena y la cordillera de Guanacaste, iniciativas que dieron como resultado muchas recolecciones de plantas en sitios no explorados del todo, o poco explorados previamente.

Como todo proyecto, el inicio fue difícil, en especial porque se tuvo que lidiar con un traslado del herbario nacional del Museo Nacional al naciente INBio en 1989. De alguna manera, esto aceleró el proyecto, así como los contactos con numerosos especialistas, para que visitaran el país.

Fue así como, con el apoyo de los diferentes profesionales en Botánica, las colecciones y la información empezaron a acopiarse. La información de cada una de las plantas recolectadas había que digitalizarla e incluirla en las bases de datos, ya fuera la localidad, las coordenadas geográficas y la altitud, así como datos sobre la especie, como su hábito, flores y frutos, al igual que los sitios donde crece. Cecilia Herrera Mora ya había sido contratada en 1987 como digitadora oficial, para generar las etiquetas de todos los duplicados, para proceder con su identificación; ella laboró durante todo el proyecto, y al final casi se convirtió en una coordinadora de este y, de hecho, en coeditora de la obra. Asimismo, cuando la sede estuvo en el INBio, también colaboró la bióloga Cristina Formoso Ohlsson, quien estuvo pocos años.

Algunas de las especies descritas en el Manual iban a contar además con una ilustración, por lo que se contrató para este trabajo tan especializado a la dibujante Silvia Troyo Jiménez, quien trazó la mayoría de las ilustraciones científicas que acompañan los diferentes volúmenes del Manual. También colaboraron de manera importante en esta función los para-taxónomos Álvaro Fernández Zúñiga (en varias familias) y Francisco Quesada Quesada (en la familia Arecaceae). Algunas ilustraciones que faltaban para los últimos volúmenes fueron ejecutadas por

\begin{tabular}{|c|c|c|}
\hline 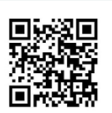 & (c) (1) (5) () & 273 \\
\hline
\end{tabular}




\section{Revista de CIENCIAS AMBIENTALES Tropical Journal of Environmental Sciences}

Revista de Ciencias Ambientales (Trop J Environ Sci)

e-ISSN: 2215-3896

(Enero-Junio, 2022) . Vol 56(1): 268-283

DOI: https://doi.org/10.15359/rca.56-1.14

Open Access: www.revistas.una.ac.cr/ambientales e-mail: revista.ambientales@una.ac.cr

Jiménez Q.

el ilustrador José Alejandro Herrera. En total, aparte de varias ilustraciones tomadas de obras antiguas y de otros países, las ilustraciones de 7 dibujantes -6 de ellos costarricenses-, complementan la prosa taxonómica de los 8 volúmenes del Manual.

\section{El grupo de para-taxónomos}

Con la creación del INBio, en 1989, cuyo objetivo se centraba en la exploración, el conocimiento, la conservación y la utilización de la biodiversidad de Costa Rica, y con la brillante idea del Dr. Daniel H. Janzen, se consolidó lo que se dio en llamar los para-taxónomos, un grupo de jóvenes rurales, algunos funcionarios guardaparques del Ministerio de Ambiente, con el objetivo de ser adiestrados en la recolección de plantas e insectos en muchos sitios del país. Fue así como, con el apoyo de diversos especialistas —incluyendo algunos botánicos-, unidos a los funcionarios de planta del Departamento de Botánica del INBio, se inició su adiestramiento.

Todo era nuevo para este grupo de parataxónomos. La gran mayoría estaban arraigados al campo, al bosque en sus sitios de origen, o trabajaban en un parque nacional, pero con casi ningún conocimiento en taxonomía ni nomenclatura vegetal. Así que, en un país tan diverso como el nuestro..., ¡había que correr a aprender!

Algunos de ellos lo hicieron muy bien, o se especializaron más en plantas, entre quienes destacaron Reinaldo Aguilar Fernández, Rodolfo Zúñiga Villegas, Gerardo Rivera Elizondo, Roberto Espinoza Retana, Ulises Chavarría García, Villy Gerardo Gamboa Romero, Flor Araya Mena, Álvaro Fernández Zúñiga, Francisco Quesada Quesada y Evelio Alfaro Vindas. Al final, Aguilar (Figura 1) decidió quedarse viviendo de la península de Osa y explorar los extraordinarios bosques de la zona, así como colaborar con entidades como el Centro de Diversidad de Plantas de la Península de Osa; cabe acotar que el registro fotográfico que tiene él sobre la flora de esta zona es realmente impresionante.

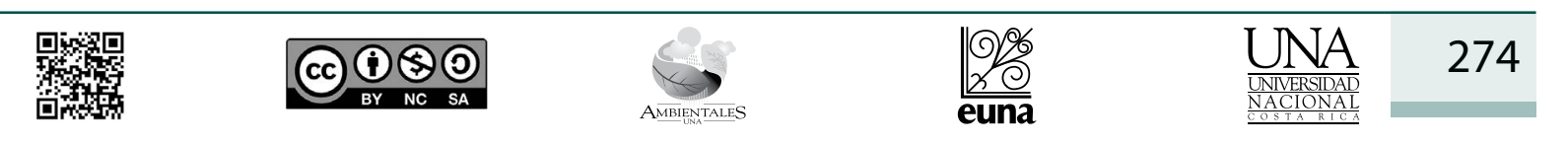




\section{Revista de CIENCIAS AMBIENTALES Tropical Journal of Environmental Sciences}

Revista de Ciencias Ambientales (Trop J Environ Sci)

e-ISSN: 2215-3896

(Enero-Junio, 2022) . Vol 56(1): 268-283

DOI: https://doi.org/10.15359/rca.56-1.14

Open Access: www.revistas.una.ac.cr/ambientales e-mail: revista.ambientales@una.ac.cr Jiménez Q.

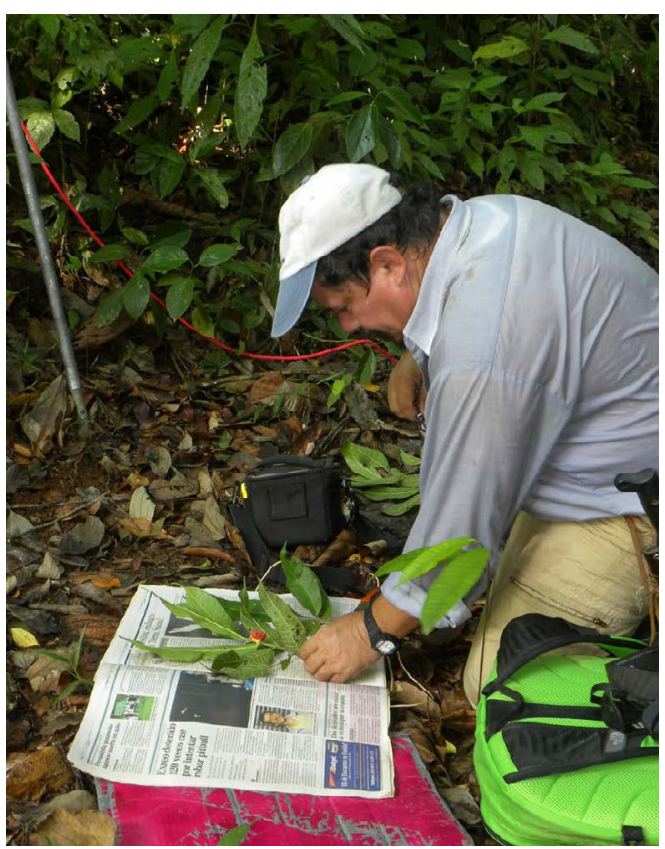

Figura 1. Reinaldo Aguilar en labores de recolección de plantas. Fuente: Reinaldo Aguilar.

Figure 1. Reinaldo Aguilar in plant collection work. Source: Reinaldo Aguilar.

\section{Otros recolectores}

Gracias a las becas otorgadas por la NSF, para agilizar el inventario nacional, el proyecto del Manual contrató a gran cantidad de profesionales en Biología y Botánica, como recolectores de plantas. Fue así como se reclutó al biólogo Gerardo Herrera Chacón, conocido como Tatica, con gran experiencia en este campo, pues había trabajado varios años con el célebre botánico Luis Diego Gómez Pignataro, cuando era director del Museo Nacional de Costa Rica. Entre las giras previas realizadas por Gerardo, cuyas recolecciones fueron importantes para el Manual, destacan la realizada al Cerro Kámuk en 1984, en compañía de Michael Grayum y Gerrit Davidse, llevando como baquiano a Salomón Romero Fallas, gran conocedor de la cordillera de Talamanca. Otros recolectores fueron Christopher Kernan, para recolectar en el Parque Nacional Corcovado; Rafael Robles González, para hacerlo en el Parque Nacional Tortuguero; Abelardo Chacón Gamboa, quien estuvo radicado en la región Caribe; Juan Marín Alpízar, en la península de Osa; y Orlando Vargas Ramírez, en la Estación Biológica La Selva, en Sarapiquí.

Es de resaltar el trabajo de Gerardo Herrera, quien con el paso del tiempo se convirtió en uno de los centroamericanos más valiosos en la recolección de plantas en las décadas de 1980 y 1990. Su carisma y conocimiento eran innegables. No era un simple recolector de plantas, sino

\begin{tabular}{|c|c|c|}
\hline 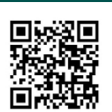 & 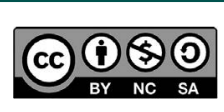 & 275 \\
\hline
\end{tabular}




\section{Revista de CIENCIAS AMBIENTALES Tropical Journal of Environmental Sciences}

Revista de Ciencias Ambientales (Trop J Environ Sci)

e-ISSN: 2215-3896

(Enero-Junio, 2022) . Vol 56(1): 268-283

DOI: https://doi.org/10.15359/rca.56-1.14

Open Access: www.revistas.una.ac.cr/ambientales e-mail: revista.ambientales@una.ac.cr

Jiménez Q.

que tenía una gran paciencia para curar las colecciones, así como para tomar las notas de campo requeridas para cada planta.

Aún recordamos verlo en Fila Matama, en el sector Atlántico de la cordillera de Talamanca, o en la península de Osa, escalando un árbol con gran agilidad y quedarse todo un día recolectando no solo en el árbol, sino que también en los árboles y otras plantas cercanas y sus epífitas. “¡Qué manera! ¡Aquí me quedo!” — decía-, pues “un buen recolector hace entre 40 y 50 números en un día”. Ese era Gerardo, a quien además se le han dedicado varias especies nuevas para la ciencia, pues sus primeras colecciones estuvieron en sus prodigiosas manos. Entre ellas figuran Bauhinia herrerae, Festuca herrerae, Gamanthera herrerae, Inga herrerae, Macrolobium herrerae, Mapania herrerae, Peperomia gerardoi, Piper gerardoi y Dicranopygium tatica (el epíteto alude a su sobrenombre de Tatica). Incluso, un género de orquídea lleva su nombre: Gerardoa. En total, la base de datos trópicos (del Jardín Botánico de Missouri) contiene más de 16.000 muestras de plantas con Gerardo como su recolector principal o como acompañante, mientras que en el Museo Nacional hay más de 12 mil colecciones suyas, como recolector principal. Sin duda, un legado impresionante de tan insigne recolector.

\section{Las personas costarricenses profesionales en Botánica}

Privilegio, es la palabra que se debe usar para agradecer al proyecto del Manual la posibilidad de colaborar en la revisión taxonómica y la redacción referida a una o varias de las familias de plantas incluidas en esta obra.

Algunas personas profesionales en Botánica tuvieron más tiempo, pues se dedicaban al $100 \%$ a esta actividad, en tanto que otras lo hicieron mientras que laboraban para otra institución. Lo importante es que existió confianza de parte de los editores del Manual.

A continuación, aparecen los nombres de los botánicos costarricenses que colaboraron en la redacción de la información acerca de una o varias familias de plantas, en diversos volúmenes del Manual. Por su cantidad de escritos, entre ellos sobresalieron Francisco Morales Quirós, Nelson Zamora Villalobos, Jorge Gómez-Laurito (†), Alexánder Rodríguez González, José González Ramírez y Quírico Jiménez Madrigal.

Además, acerca de una sola o unas pocas familias, escribieron Armando Estrada Chavarría, Silvana Martén Rodríguez, Ricardo Kriebel Haehner, Joaquín Sánchez González, Mario Blanco Coto, José Esteban Jiménez Vargas, Luis Jorge Poveda Álvarez, Armando Soto Solís, Cristina Formoso Ohlsson, Luis González Arce, Eduardo Chacón Madrigal, Gina Umaña Dodero y Daniel Santamaría Aguilar. Cabe señalar que algunos botánicos incluso unieron esfuerzos, y juntos publicaron una o varias familias, como sucedió con Quírico Jiménez y Francisco Morales con la familia Bignoniaceae, al igual que José González y Luis Jorge Poveda con la familia Lauraceae, entre otros.

Es pertinente resaltar que los diversos borradores o manuscritos para cada familia eran revisados por Hammel, Grayum y Cecilia Herrera, quienes estaban inmersos a tiempo completo

\begin{tabular}{|c|c|c|}
\hline 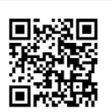 & (c) (i) (5) () & 276 \\
\hline
\end{tabular}




\section{Revista de CIENCIAS AMBIENTALES Tropical Journal of Environmental Sciences}

Revista de Ciencias Ambientales (Trop J Environ Sci)

e-ISSN: 2215-3896

(Enero-Junio, 2022) . Vol 56(1): 268-283

DOI: https://doi.org/10.15359/rca.56-1.14

Open Access: www.revistas.una.ac.cr/ambientales e-mail: revista.ambientales@una.ac.cr

Jiménez Q.

a esta actividad, además de que los dos primeros también estaban dedicados a la taxonomía de algunas de las familias en las cuales eran especialistas, o llegaron a serlo.

Cabe señalar que hubo familias que, por su cantidad de especies, o debido a las pocas revisiones previas de especialistas, resultaron más complicadas de tratar. Tal es el caso de la familia Asteraceae, que incluye 418 especies, pertenecientes a 161 géneros, la cual fue estudiada por Alexánder Rodríguez, para lo cual antes tuvo que aprender sobre su morfología, a veces con poca literatura disponible, y con géneros complicados, como Ageratina. Según sus propias palabras "aún sigo aprendiendo y entendiendo sobre esta complicada familia".

Otras familias complicadas y grandes, tratadas por costarricenses, fueron Bromeliaceae (195 especies, en 17 géneros), Poaceae (488 especies, en 139 géneros) y Fabaceae (593 especies, en 136 géneros), a cargo de Francisco Morales, las 2 primeras, y de Nelson Zamora la última.

\section{Sitios de recolección y cantidad de especímenes}

Una estrategia por parte del proyecto del Manual fue realizar giras o expediciones a sitios menos explorados, como las zonas intermedias del Caribe en la cordillera de Talamanca. Sin embargo, finalmente se sumaron otros sitios, como los parques nacionales Braulio Carrillo, Corcovado, Rincón de la Vieja, Santa Rosa y Tortuguero, así como las regiones de Monteverde y Carara (Jiménez \& Grayum, 2002), además de otra serie de localidades de gran importancia, en sitios no protegidos, como el cerro Turrubares, el cerro Caraigres, y la vertiente Caribe del volcán Orosí.

Según Hammel et al. (2004), el contenido del Manual está basado en cerca de 300.000 especímenes, sin contar las muestras repetidas (duplicados), a menudo recogidas para compartir entre herbarios. Cabe acotar que, con la desaparición de las actividades botánicas en el INBio, hace pocos años, las colecciones de su herbario (INB) pasaron a ser propiedad del Herbario Nacional de Costa Rica (CR), por lo que es posible que, del trabajo desarrollado por muchos recolectores en la época del proyecto del Manual, en este herbario debe haber hoy un mínimo de dos especímenes de la gran mayoría de cada una de las especies recolectadas para el proyecto del Manual.

Afortunadamente, durante el período de exploración, algunos de los botánicos y futuros autores de otros países lograron visitar Costa Rica para colaborar en la exploración botánica, y a su vez revisar los especímenes, tanto los recientes como los conservados en los herbarios del país. Cuando fue posible, los duplicados de muchas de las especies recolectadas durante el proyecto fueron enviados al Jardín Botánico de Missouri, para de allí hacerlas llegar a los especialistas extranjeros no residentes en Costa Rica. En el mejor de los casos, también se logró dejar un duplicado depositado en Missouri, para futuros estudios. Además, es importante mencionar la visita de botánicos costarricenses a Missouri, para trabajar taxonómicamente sus familias. En fin, el periodo de exploración fue en gran medida el apogeo del proyecto en cuanto a intercambio de especialistas, de muestras botánicas y, por ende, un periodo de muchísimo ánimo y actividad en los herbarios.

\begin{tabular}{|c|c|c|c|c|c|}
\hline 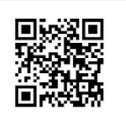 & (c) (1) (5) () & $\theta_{\text {AMEIENTIISS }}$ & $\frac{O \%}{2)}$ & $\frac{\text { UNA }}{\frac{\text { UNIVERSIDAD }}{\text { NACIONAL }}}$ & 277 \\
\hline
\end{tabular}




\section{Revista de CIENCIAS AMBIENTALES Tropical Journal of Environmental Sciences}

Revista de Ciencias Ambientales (Trop J Environ Sci)

e-ISSN: 2215-3896

(Enero-Junio, 2022) . Vol 56(1): 268-283

DOI: https://doi.org/10.15359/rca.56-1.14

Open Access: www.revistas.una.ac.cr/ambientales e-mail: revista.ambientales@una.ac.cr

Jiménez Q.

\section{Resultados y logros extraordinarios}

Hablar de los resultados y los logros en la exploración botánica de Costa Rica a través del tiempo, es pasar de relativamente pocas especies en el pasado, a muchas en la actualidad, y quizá nunca se tenga un número totalmente exacto de estas. Lo que sí está claro es que, en un poco más de las tres décadas que duró el proyecto del Manual, culminado con la publicación del último volumen a finales del 2020 (Figura 2), estos son los resultados más importantes:

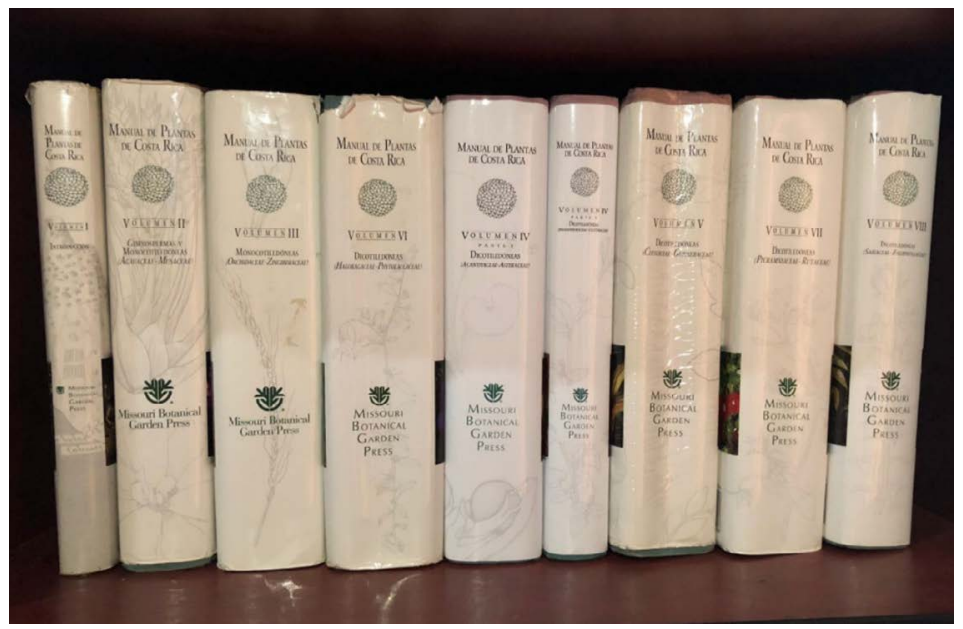

Figura 2. Los ocho volúmenes del Manual.

Figure 2. The eight volumes of the Manual.

a) Hoy se conocen más de 11000 especies de plantas, incluyendo los helechos, los cuales no fueron tratados en el Manual.

b) En el momento de la publicación del primer volumen del Manual, Hammel et al. (2004) acotaban que desde 1993 hasta esa fecha se habían descrito y publicaron 209 especies nuevas de orquídeas, y que desde 1989 al 2000 a la flora de Costa Rica se le agregaron más de 500 especies de plantas vasculares, incluyendo unos 200 registros nuevos para el país de especies previamente descritas. Hoy, casi dos décadas después, estas cifras se han superado con creces, como resultado de especímenes sin identificar enviados a una gran cantidad de especialistas en diversas familias, tanto de varios países del continente americano como europeo, al igual que de las especies publicadas por botánicos nacionales.

c) Los árboles han sido un grupo bastante estudiado en el país. Aun así, sorprende que, de manera esporádica, aparezcan especies nuevas, pertenecientes a géneros desconocidos, o convertidos en nuevos reportes para el país. Por ejemplo, entre las especies más relevantes descubiertas, sobresale el árbol Ticodendron incognitum de la familia Ticodendraceae

\begin{tabular}{|c|c|c|}
\hline 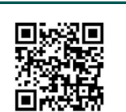 & (c) $\underset{\mathrm{BY}}{(1)}\left(\begin{array}{l}\mathrm{NC} \\
\mathrm{SA}\end{array}\right.$ & 278 \\
\hline
\end{tabular}




\section{Revista de CIENCIAS AMBIENTALES Tropical Journal of Environmental Sciences}

Revista de Ciencias Ambientales (Trop J Environ Sci)

e-ISSN: 2215-3896

(Enero-Junio, 2022) . Vol 56(1): 268-283

DOI: https://doi.org/10.15359/rca.56-1.14

Open Access: www.revistas.una.ac.cr/ambientales e-mail: revista.ambientales@una.ac.cr

Jiménez Q.

(Figura 3), publicada como una familia nueva por los desaparecidos colegas Luis Diego Gómez y Jorge Gómez-Laurito. Es importante mencionar sobre esta especie que la misma había sido herborizada desde hacía varios años, y que sus colecciones estuvieron ubicadas dentro de otras familias. No obstante, después de su descripción como una nueva especie, se supo que, en realidad, tiene una amplia distribución, pues está presente en casi todos los países desde México hasta Panamá.

d) El descubrimiento de especies nuevas ha traído alegría a los botánicos, así como también el descubrimiento y publicación de nuevos géneros, o de géneros conocidos en otros países o continentes, y que se convirtieron en nuevos reportes para el país. Destacan entre ellos palmas como Aiphanes y Colpothrinax, o plantas herbáceas como Caladium, Pacourina, Tetranema, Thoracocarpus y Lennoa.

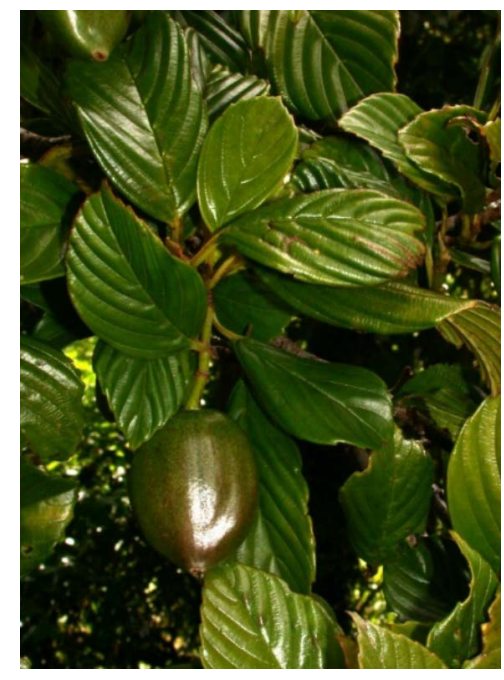

Figura 3. Ticodendron incognitum con frutos. Fuente: Barry Hammel. Figure 3. Ticodendron incognitum with fruits. Source: Barry Hammel.

e) Se conocen cerca de 2500 especies de árboles en nuestro país, algunos bien estudiados y documentados, porque muchos de ellos han sido muy utilizados por su madera. En este contexto, el Cuadro 1 resume algunos géneros de árboles que se pueden destacar por ser de otros continentes, de origen suramericano, o endémicos, los cuales fueron recolectados por primera vez en Costa Rica, gracias al proyecto del Manual.

\begin{tabular}{|c|c|c|}
\hline 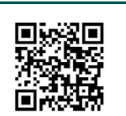 & (c) $\underset{\mathrm{BY}}{(1)} \mathrm{NC}_{\mathrm{NA}}(0)$ & 279 \\
\hline
\end{tabular}




\section{Revista de CIENCIAS AMBIENTALES Tropical Journal of Environmental Sciences}

Revista de Ciencias Ambientales (Trop J Environ Sci) e-ISSN: 2215-3896

(Enero-Junio, 2022) . Vol 56(1): 268-283

DOI: https://doi.org/10.15359/rca.56-1.14 Open Access: www.revistas.una.ac.cr/ambientales e-mail: revista.ambientales@una.ac.cr

Jiménez Q.

Cuadro 1. Algunos géneros de árboles recolectados por primera vez en Costa Rica durante el proyecto del Manual de Plantas.

Table 1. Some tree genera collected for the first time in Costa Rica during Manual de Plantas de Costa Rica project.

\begin{tabular}{|c|c|c|}
\hline Nombre & Familia & Importancia \\
\hline Caryodaphnopsis & Lauraceae & $\begin{array}{l}\text { Género asiático y de Suramérica. Una especie en Costa Rica, } C \text {. } \\
\text { burgeri, dedicada al botánico William Burger. }\end{array}$ \\
\hline Dacryodes & Burseraceae & $\begin{array}{l}\text { Género presente en África, Asia y Suramérica. En Costa Rica se } \\
\text { conoce la especie talamancensis. }\end{array}$ \\
\hline Endlicheria & Lauraceae & Género de Suramérica. En Costa Rica se conoce la especie formosa. \\
\hline Gamanthera & Lauraceae & $\begin{array}{l}\text { Género y especie nuevos, así como endémicos de Costa Rica. Se } \\
\text { conoce la especie herrerae. }\end{array}$ \\
\hline Lubaria & Rutaceae & Género de Suramérica. En Costa Rica se conoce la especie aroensis. \\
\hline Nyssa & Nyssaceae & $\begin{array}{l}\text { Género del sureste de EE.UU. y el sureste asiático. En Costa Rica se } \\
\text { conoce la especie talamancana, presente aquí y en Panamá. }\end{array}$ \\
\hline Paramachaerium & Fabaceae & $\begin{array}{l}\text { Género de Suramérica. En Costa Rica y Panamá se conoce la especie } \\
\text { gruberi. }\end{array}$ \\
\hline Pleodendron & Canellaceae & $\begin{array}{l}\text { Género y familia como nuevo reporte para el país. En Costa Rica se } \\
\text { conoce la especie endémica costaricense. }\end{array}$ \\
\hline Pradosia & Sapotaceae & $\begin{array}{l}\text { Género de Suramérica. En Costa Rica se conoce la especie } \\
\text { atroviolacea. }\end{array}$ \\
\hline Raputiarana & Rutaceae & $\begin{array}{l}\text { Género de Suramérica. En Costa Rica se conoce la especie } \\
\text { heptaphylla. }\end{array}$ \\
\hline Ruptiliocarpon & Lepidobotryaceae & $\begin{array}{l}\text { Género descrito primero en Corta Rica, donde se conoce la especie } \\
\text { llamada "caracolito". Ahora también se conoce en Panamá y Sur } \\
\text { América. }\end{array}$ \\
\hline Stephanopodium & Dichapetalaceae & $\begin{array}{l}\text { Género presente desde Nicaragua hasta Suramérica. En Costa Rica } \\
\text { se conoce la especie costaricense. }\end{array}$ \\
\hline Povedadaphne & Lauraceae & $\begin{array}{l}\text { Género nuevo para Costa Rica, dedicado al botánico Luis Poveda. } \\
\text { En Costa Rica se conoce la nueva especie quadriporata. }\end{array}$ \\
\hline
\end{tabular}

f) El Manual reconoce para el país un total de 237 familias de plantas con semilla (o sea, sin considerar los helechos, los musgos y las algas). De ellas, las que tienen la mayor cantidad de especies de árboles son Fabaceae (262), Rubiaceae (139), Lauraceae (110), Melastomataceae (88) y Moracaeae (65). Se conoce un total de 2034 géneros, de los cuales los que tienen la mayor cantidad de árboles son Inga (54), Miconia (50), Ocotea (45), Ficus (44), Pouteria (40), Lonchocarpus (32), Eugenia (28), Sloanea (27) y Nectandra (20).

g) De todas las familias de plantas con semillas, Orchidaceae es la que tiene la mayor cantidad de especies, con 1318 especies en 174 géneros (Dressler, 2003), aunque más recientemente Bogarín et al. (2016) han incrementado esta cifra hasta 1574 especies. Sin embargo, con seguridad hay más de 1600 especies (con un alto grado de endemismo) y con gran cantidad

\begin{tabular}{|c|c|c|c|c|}
\hline 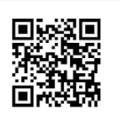 & (c) (1) (9) & $\underset{\text { AMBIENTAES }}{\Leftrightarrow}$ & $\frac{10 \%}{\frac{9}{2}}$ & 280 \\
\hline
\end{tabular}




\section{Revista de CIENCIAS AMBIENTALES Tropical Journal of Environmental Sciences}

Revista de Ciencias Ambientales (Trop J Environ Sci)

e-ISSN: 2215-3896

(Enero-Junio, 2022) . Vol 56(1): 268-283

DOI: https://doi.org/10.15359/rca.56-1.14

Open Access: www.revistas.una.ac.cr/ambientales e-mail: revista.ambientales@una.ac.cr

Jiménez Q.

de cambios genéricos, debido a refinados estudios moleculares. Además, como es usual, en los países donde se cuenta con especialistas en alguna familia, los estudios son más profundos y detallados, lo cual favorece la detección de nuevas especies. En tal sentido, se puede afirmar que Costa Rica es meca de la orquideología, gracias a las labores del Jardín Botánico Lankester, de la Universidad de Costa Rica, especializado en la sistemática vegetal y ecología de las orquídeas.

h) Otras familias con gran cantidad de especies son Fabaceae (593, en 136 géneros), Poaceae (488, en 139 géneros), Piperaceae (465, en tres géneros), Rubiaceae (458, en 89 géneros, Asteraceae (418, en 161 géneros), Melastomataceae (303, en 34 géneros) y Araceae (249, en 20 géneros).

i) Es importante rescatar la importancia del endemismo. Mesoamérica es una de las nueve regiones del planeta con una alta diversidad de especies endémicas, con 5000 de ellas, es decir un $1.7 \%$ del total de las plantas conocidas en el planeta. En tal sentido, Costa Rica contribuye con unas 1000 especies endémicas. Las familias con mayor endemismo son Acanthaceae, Bromeliaceae, Lauraceae y Marantaceae, pero ninguna sobrepasa a Orchidaceae, pues de las 1574 de especies registradas hasta ahora en el país (Bogarín et al., 2016), 485 (38 \%) son endémicas.

\section{Consideraciones finales}

No hay duda de que el Manual de Plantas de Costa Rica es una obra de inmenso valor científico. En ella queda plasmada no solo la información de la diversidad de las plantas de Costa Rica, sino que también el ejemplo de colaboración que existió entre tres grandes instituciones: el Jardín Botánico de Missouri, el INBio y el Museo Nacional de Costa Rica, con el apoyo de una red nacional e internacional de especialistas en botánica, interesados en estudiar y aprender sobre nuestra riqueza florística.

Asimismo, debe resaltarse que la conclusión del Manual, materializado en ocho volúmenes ricos en información, representa solamente el final del proyecto, pero no será el final del estudio de nuestra flora, pues todavía tenemos mucho que aprender y documentar sobre el maravilloso reino de las plantas, y la forma en que se manifiesta en Costa Rica, que es una pequeña pero asombrosa región del trópico americano.

Este Manual, sin duda, será una guía que debe ser tomada en cuenta para apreciar, estudiar, conocer y proteger un grupo tan importante de esta biodiversidad como son nuestras plantas. Su conservación y uso responsable será un legado de gran importancia para las futuras generaciones, pues nuestra diversidad florística debe seguirnos acompañando por siempre. No debemos permitir que se pierda ninguna de nuestras especies.

\begin{tabular}{|c|c|c|}
\hline 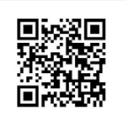 & (c) (i) (5) () & 281 \\
\hline
\end{tabular}




\section{Revista de CIENCIAS AMBIENTALES Tropical Journal of Environmental Sciences}

Revista de Ciencias Ambientales (Trop J Environ Sci) e-ISSN: 2215-3896 (Enero-Junio, 2022) . Vol 56(1): 268-283 DOI: https://doi.org/10.15359/rca.56-1.14 Open Access: www.revistas.una.ac.cr/ambientales e-mail: revista.ambientales@una.ac.cr Jiménez Q.

\section{Agradecimientos}

Un agradecimiento especial a los amigos y colegas Barry Hammel y Michael Grayum, por la revisión del manuscrito, así como por sus ideas y comentarios para mejorarlo. A Gerardo Herrera Chacón y Cecilia Herrera Mora por la valiosa información brindada. A mi hijo César Fabián Jiménez Vargas, por colaborar en la edición del artículo, y al colega Luko Hilje por sus observaciones al manuscrito original.

\section{Referencias}

Alvarado, G. E., \& Cárdenas, G. (2016). Geology, tectonics, and geomorphology of Costa Rica: A natural history approach. En M. Kappelle (Ed.), Costa Rican Ecosystems (pp. 30-63). University of Chicago Press. https://doi.org/10.7208/chicago/9780226121642.003.0003

Bogarín D., Pupulin, F., Smerts, E., \& Gravendeel, B. (2016). Evolutionary diversification and historical biogeography of the Orchidaceae in Central America with emphasis on Costa Rica and Panama. Lankesteriana 16(2), 189-200. https://doi.org/10.15517/lank.v16i2.26005

Burger, W. C. (1980). Why are so many kinds of flowering plants in Costa Rica? Brenesia, 31(8), 71-88. https://doi.org/10.2307/1308218

Dressler, R. (2003). Orchidacaeae. En B. E. Hammel, M. H. Grayum, C. Herrera \& N. Zamora (Eds), Manual de Plantas de Costa Rica. (Vol. III, pp. 1-596). Missouru Botanical Garden Press.

Gámez Lobo, R., León Azofeifa, P., \& Hilje Quirós, L. (2021). La biodiversidad de Costa Rica en dos siglos de vida independiente, y una mirada hacia el tricentenario. Revista del Archivo Nacional de Costa Rica, 85, 1-44. http://dgan.go.cr/ran/index.php/RAN/article/view/529

Gómez, L. D. (1986). Vegetación de Costa Rica, Apuntes para una Biogeografía costarricense. (Vol. 1). Editorial Universidad Estatal a Distancia

Grayum, M. H. (1991). Botanical exploration of the eastern Cordillera de Talamanca, Costa Rica. Res. \& Explor, 7(4), 495-496.

Hammel, B. E., Grayum, M. H., Herrera C., \& Zamora, N. (Eds.) (2004). Manual de Plantas de Costa Rica. (Vol. I.). Missouru Botanical Garden Press.

Hilje Quirós, L. (2013). Trópico agreste; la huella de los naturalistas alemanes en la Costa Rica del siglo XIX. Editorial Tecnológica de Costa Rica.

Jiménez, Q. (1999). Consideraciones para el manejo y conservación de 18 especies forestales vedadas en Costa Rica. Guaiacum sanctum L. (Guayacán real) un caso particular de estudio (Tesis de Maestría, Universidad Internacional de Andalucía), España.

\begin{tabular}{|c|c|c|c|c|c|}
\hline 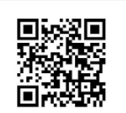 & (c) (i) (5) () & $\theta_{\text {AMBIENTALS }}^{6}$ & $\frac{10 \%}{\frac{9}{2}}$ & $\frac{\text { UNA }}{\frac{\text { UNIVERSIDAD }}{\text { UNACIONAL }}}$ & 282 \\
\hline
\end{tabular}




\section{Revista de CIENCIAS AMBIENTALES

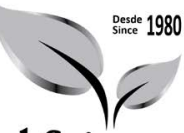 Tropical Journal of Environmental Sciences}

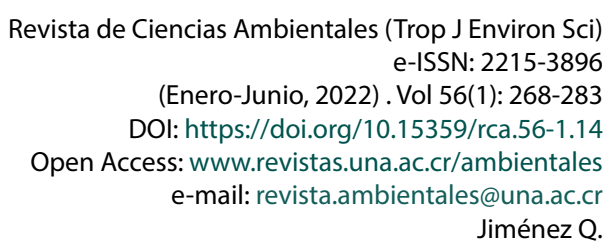

Jiménez, Q., \& M. H. Grayum. (2002). Vegetación del Parque Nacional Carara, Costa Rica. Brenesia, 57(58), 25-66. https://docplayer.es/14718139-Vegetacion-del-parque-nacional-carara-costa-rica.html

Kappelle, M. (2016). Setting the Stage. Costa Rican Ecosystems. (Chapter 1). 3-16.

León, J. (2002). La exploración botánica de Costa Rica en el siglo XIX. En: Ciencia y técnica en la Costa Rica del siglo XIX. G. Peraldo (ed.). Editorial Tecnológica de Costa Rica.

Obando Acuña, V. (2002). Biodiversidad en Costa Rica: Estado del Conocimiento y Gestión. Instituto Nacional de Biodiversidad.

Ulloa, C., Acevedo-Rodríguez, P., Beck, S., Belgrano, M., Bernal, R., Berry, P., Brako, L., Celis, M., Davidse, G., Forzza,R., Gradstein, R., Hokche, O., León, B., León-Yánez,S., Magill,R., Neill, D., Nee, M., Raven, P., Stimmel, H... Jørgensen, P. (2017). Integrated assessment of the vascular plant species of the Americas. Science, 358, 1614-1617. https://doi.org/10.1126/ science.aao0398

Valerio, C. E. (2006). Costa Rica: ambiente y biodiversidad. Editorial Instituto Nacional de Biodiversidad (INBio)

\begin{tabular}{|c|c|c|}
\hline 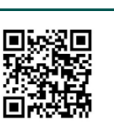 & (c) (1) () () (2) & 283 \\
\hline
\end{tabular}

